\section{Área de cuentas oficinas BBDO Chile} Santiago, Chile
La habilitación de una franja longitudinal de una planta libre en un edificio existente, destinada a las áreas de cuentas de las oficinas de una agencia de publicidad, se resuelve a partir de un muro-mueble móvil que recorre toda la extensión del área intervenida. El almacenamiento ordena y define el lugar de trabajo.
The refurbishing of a longitudinal strip on an open floor of an existing building, destined to the accounts area of the offices of a publicity agency, is resolved by means of a mobile furniture-wall that is used along the entire extension of the refurbished area. Storage orders and defines the work area.

Álvaro Benítez Arquitecto, Pontificia Universidad Católica de Chile

Emilio de la Cerda Profesor Instructor, Escuela de Arquitectura, Pontificia Universidad Católica de Chile

Tomás Folch Profesor Instructor, Escuela de Arquitectura, Pontificia Universidad Católica de Chile

Daniel Rosenberg Profesor Instructor, Escuela de Arquitectura, Pontificia Universidad Católica de Chile

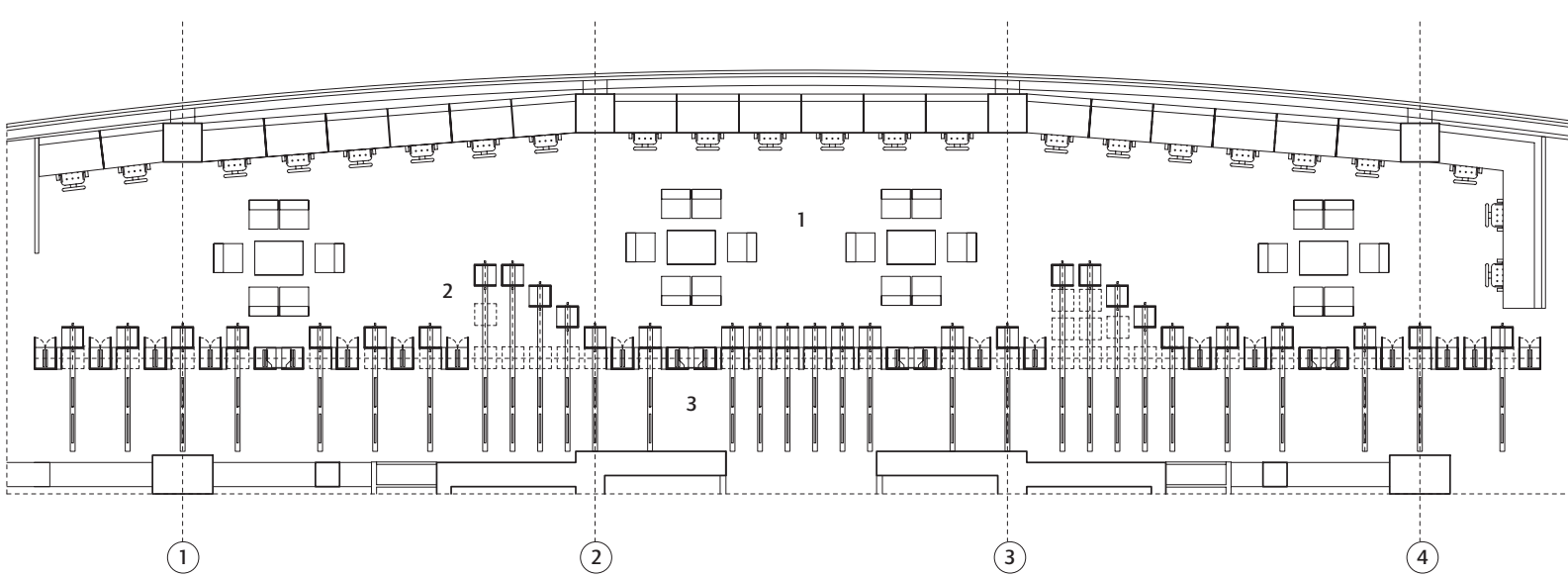

PLANTA

1 Zona de trabajo

2 Muro mueble

3 Circulación principal

En una planta libre el espacio finalmente se divide de acuerdo a las necesidades específicas de cada oficina, perdiéndose la cualidad de planta libre una vez que las divisiones se fijan, al generar oficinas y pasillos.

Para el área de cuentas de BBDO, se debía definir un límite entre la circulación principal y una zona de trabajo. La intención del proyecto fue mantener cierta libertad del espacio al fijar los límites de transformación de un elemento ambiguo. El proyecto, como mueble o muro, propone una serie de posibilidades formales que buscan devolver a la planta ciertos grados de libertad, sin sacrificar la independencia entre las distintas unidades del programa.

MURO Y MUEBLE / El proyecto tiene como objetivo inicial el separar y el guardar. Es así como se libera el espacio al juntar todo en un solo gran objeto que separa, como si fuera un muro, pero con el espesor propio de un mueble. Asimismo, se mueve como un mueble pero se encuentra amarrado a la estructura del edi- ficio al igual que un muro, lo cual limita su libertad de movimiento. Es vertical y opaco como un muro pero está conformado por módulos independientes que, como muebles, se separan del suelo, del cielo y entre sí. Esta ambigüedad constituye la característica fundamental del proyecto. Un muro-mueble grueso y móvil, un límite esponjoso que sirve para guardar y que se abre o cierra para entrar o salir.

INDEFINICIÓN FORMAL / El proyecto se construye en base a un trazado de rieles transversales a una línea recta. Sobre estos rieles es posible el cambio de posición de cada módulo afectando la lectura del total. Cada riel determina dos puntos y una medida entre estos, fijando un máximo y un mínimo de movimiento. Si bien dichos límites nos entregan dos posiciones determinadas, el resultado de la forma es incierto. Entre ambas posiciones las combinaciones y por tanto las configuraciones formales pueden ser infinitas. De esta manera $e l$ diseño de la forma sólo plantea ciertas reglas 


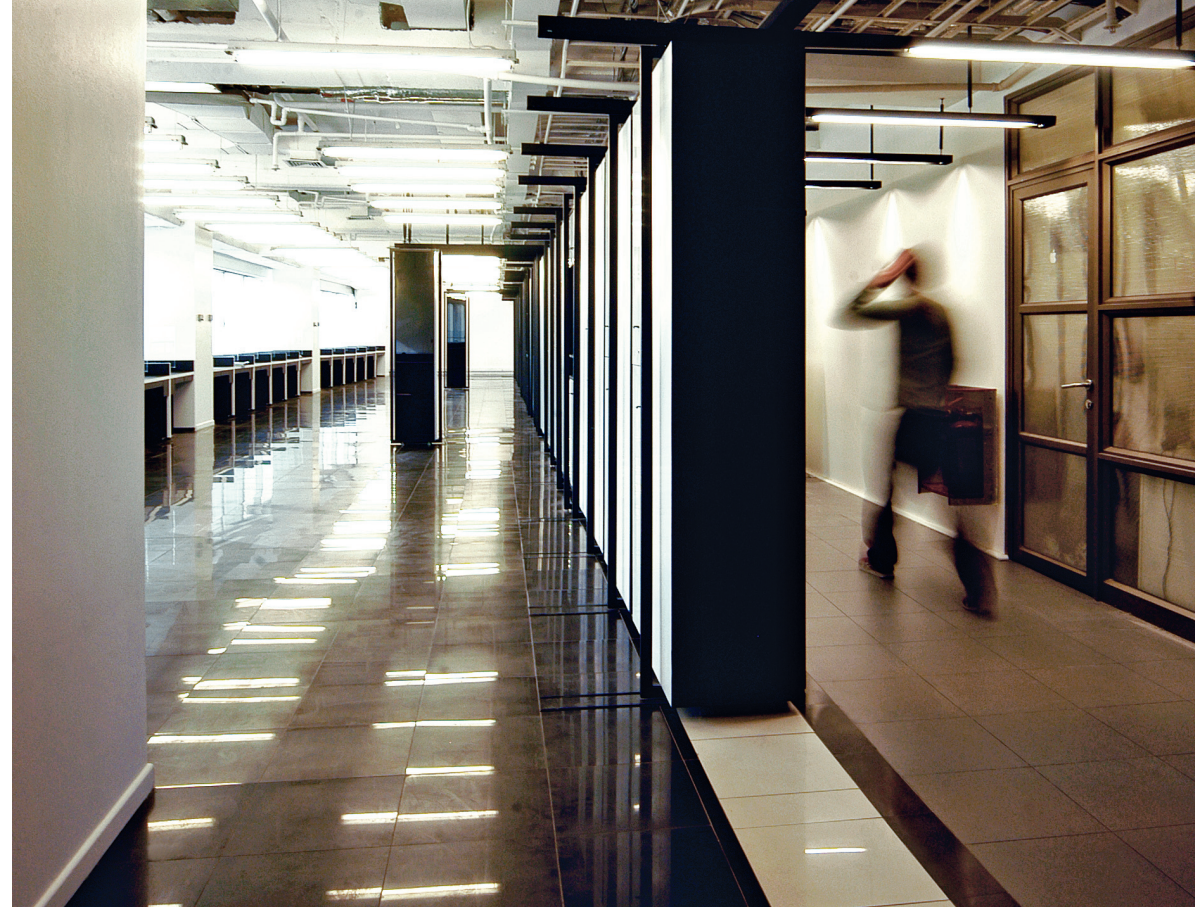

de operación al dejar el resultado final abierto a las decisiones y arbitrariedades que puedan surgir con el tiempo, necesidades o gustos.

Gran Cajón / Es posible modificar la forma de acuerdo a una globalidad y también a la necesidad de operación individual. Si un cajón de la altura de un muro se abre, su movimiento afectará la totalidad del espacio. En este caso la operación determina, además del objeto operable propiamente tal, una serie de formalizaciones adicionales que permiten su funcionamiento. Es así como aparecen rieles sobre el suelo y guías colgando del cielo. Todos estos elementos surgen de una necesidad funcional ya que cada módulo debe poder abrirse fácilmente por el usuario en el momento y las veces que estime necesario. Esta restricción técnica implica un diseño acucioso del sistema como mecanismo, por lo cual se desarrollaron modelos previos y pruebas que nos acercaran a un funcionamiento óptimo de operación. ARq

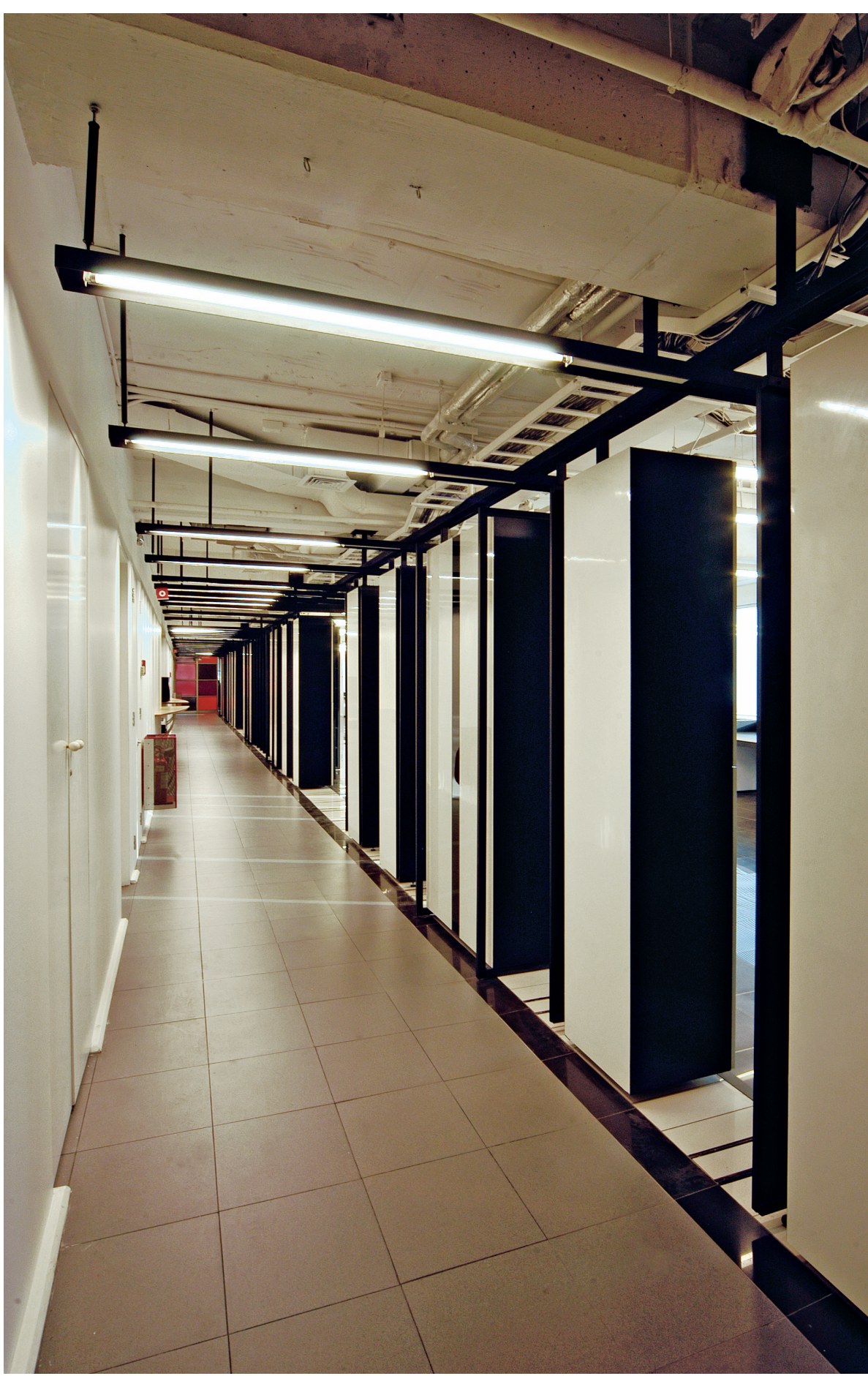



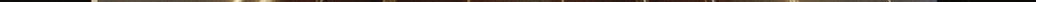

ÁREAS DE CUENTAS BBDO CHILE

Arquitectos Álvaro Benítez, Emilio de la Cerda, Tomás Folch, Daniel Rosenberg. Owar Arquitectos

Ubicación Avda. Vitacura 2939, piso 14, Vitacura, Chile Cliente BBDO Chile

Cálculo estructural Larraín Vial y Asociados

Construcción Constructora GYR Ltda. (Obras civiles)

Constructora Briceño Ltda. (Mobiliario), David Hermosilla (estructuras metálicas)

Materialidad estructura de acero, piso porcelanato, madera aglomerada enchape formica

Presupuesto 9 UF/ $\mathrm{m}^{2}$ (US\$ $313 / \mathrm{m}^{2}$ )

Superficie construida $245 \mathrm{~m}^{2}$

Año proyecto 2007

Año construcción 2007

Fotografía Álvaro Benítez, Daniel Rosenberg 


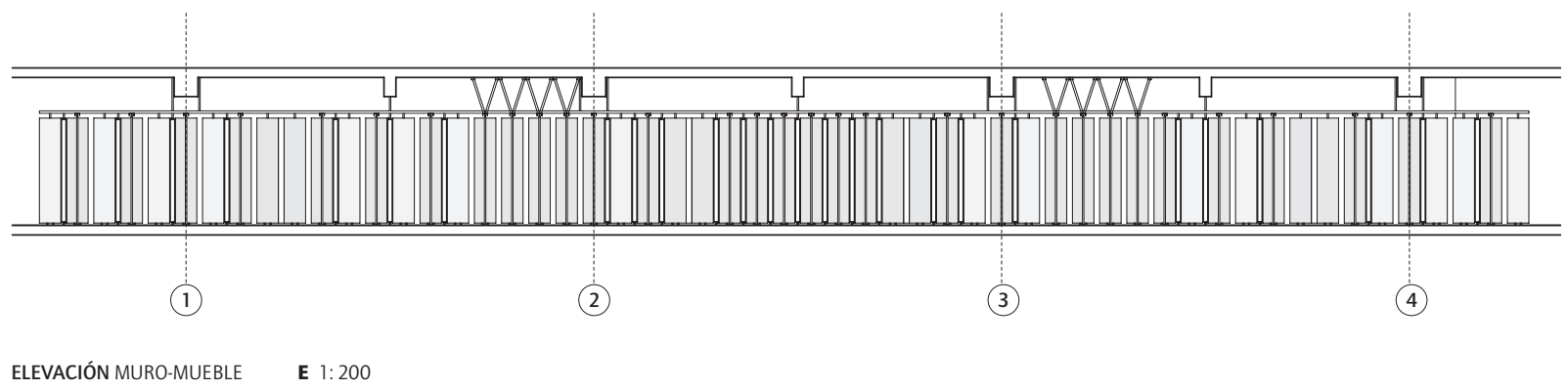

1 Amarre a losa

2 Guías estructura cielo

3 Rieles suelo

1 Fijación a losa. Ángulo Fe 50×50×3 mm +

Pletina Fe $5 \mathrm{~mm}$

2 Pasador hilo $20 \mathrm{~mm}$

3 Tubo de acero 1"1/4 $5 \mathrm{~mm}$

4 Canal riel sistema corredero

5 Espejo $3 \mathrm{~mm}$

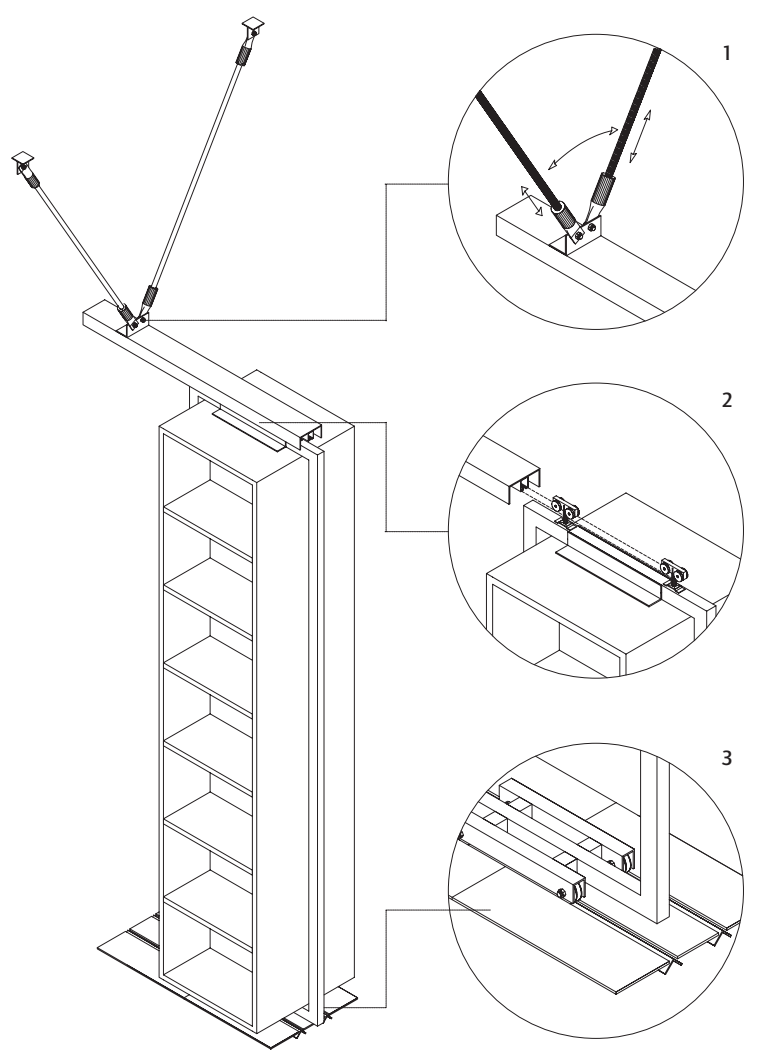

6 Pasador $10 \mathrm{~m}$

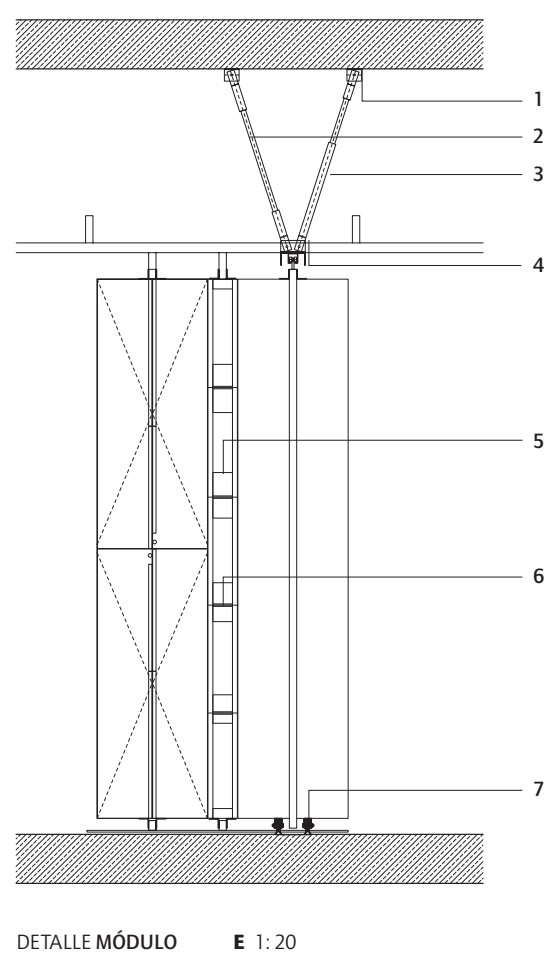

ISOMÉTRICA DETALLE MÓDULO

DETALLE MÓdULO E 1:20 